\title{
NIL ALGEBRAS SATISFYING AN IDENTITY OF DEGREE THREE
}

\author{
RAYMOND COUGHLIN
}

\begin{abstract}
Let $A$ be a nonassociative algebra over a field $F$ with a function $g: A \times A \times A \rightarrow F$ such that $(x y) z=g(x, y, z) x(y z)$ for all $x, y$, and $z$ in $A$. Algebras satisfying this identity have been studied by Michael Rich and the author. It is shown here that a finitedimensional nil power-associative algebra satisfying the above identity is nilpotent.
\end{abstract}

Let $A$ be an algebra over a field $F$ with a function $g: A \times A \times A \rightarrow F$ such that

$$
(x y) z=g(x, y, z) x(y z)
$$

for all $x, y, z$ in $A$. Semisimple algebras satisfying (1) and

$$
x^{2} x=x x^{2}
$$

have been studied by Michael Rich and the author [2]. A power-associative algebra $A$ is an algebra $A$ such that for every $x$ in $A$ the subalgebra generated by $x$ in $A$ is associative. A nil power-associative algebra is an algebra in which for every $x$ there exists an integer $n(x)$ such that $x^{n(x)}=0$. An algebra is nilpotent if there exists an integer $n$ such that $A^{n}=0$. It is shown that a finite-dimensional nil power-associative algebra satisfying (1) is nilpotent. Throughout this paper we will assume that all algebras are finite dimensional and power-associative. We define the associator $(x, y, z)=(x y) z-x(y z)$. The linearization of $(2)$ is

$$
\begin{aligned}
(x, y, z)+(y, z, x)+(z, x, y)+(x, z, y) & \\
& +(z, y, x)+(y, x, z)=0 .
\end{aligned}
$$

We define $R(x)$ and $L(x)$ to be the usual endomorphisms on $A z R(x)=z x$ and $z L(x)=x z$. We can then write (1) in the form

$$
\begin{aligned}
& z R(a) R(b)=\alpha z R(a b), \\
& z L(a) R(b)=\beta z R(b) L(a)
\end{aligned}
$$

Presented to the Society April 9, 1971; received by the editors June 22, 1971 and, in revised form, October 18, 1971.

AMS 1969 subject classifications. Primary 1720.

Key words and phrases. Power-associative, nil algebra, nilpotent algebra.

(c) American Mathematical Society 1972 
for all $z, a, b$ in $A$ and $\alpha=g(z, a, b), \beta=g(a, z, b) \in F$. Throughout we assume that the characteristic of $F \neq 2$.

We begin with

THEOREM 1. If $A$ is an algebra over $F$ satisfying (1) with $x^{N}=0$, then the ring $\mathscr{R}$ generated by the right and left multiplications by powers of $x$ is a nilpotent ring.

Proof. From (1) we can write

$$
z L\left(x^{n}\right)=\alpha z L(x)^{n}
$$

for any $z$ in $A$ and where $\alpha \in F$ depends upon $z, x$ and $n$. For any element $S$ in $\mathscr{R}$ and $z$ in $A$ we can use (5) to pass all the right multiplications to the left and then use (4) and (5) to write $z S$ as a linear combination of terms of the form $z S=\alpha z R\left(x^{m}\right) L(x)^{r}$ for some $\alpha \in F$. Now let $T \in \mathscr{R}$ and consider $T^{k}$ where $k=N+2^{N}(N+1)$. We can write $z T$ as a linear combination of terms of the form

$$
\alpha z R\left(x^{m}\right) L(x)^{r}
$$

where $r \geqq 2^{N}(N+1)$. We can choose a nonzero expression of this form such that $r$ is minimal. From (3) we have

$$
z L(x)^{2}=z L\left(x^{2}\right)+\beta_{1} z R\left(x^{2}\right)+\beta_{2} z R(x) L(x)
$$

where $\beta_{1}, \beta_{2} \in F$. Into (7) substitute (8) for the two leftmost $L(x)$ 's. By our choice of $r,(7)$ is equal to

$$
\alpha z R\left(x^{m}\right) L\left(x^{2}\right) L(x)^{r-2} \text {. }
$$

Into (9) substitute (8) again for the two leftmost $L(x)$ 's and, continuing this process, we get that (7) is equal to

$$
\alpha z R\left(x^{m}\right) L\left(x^{2}\right)^{r_{1}} I_{1}(x)
$$

when $I_{1}(x)$ is the identity operator or $L(x)$, depending upon whether $r$ is even or odd. Note that (6) allows us to still utilize the choice of $r$ and hence to conclude that $r_{1}$ is minimal. Replace $x$ by $x^{2}$ in (8) and then use this same process to write (10) in the form $\alpha z R\left(x^{m}\right) L\left(x^{4}\right)^{r_{2}} I_{2}$ where $I_{2}$ is either the identity operator, $L(x), L\left(x^{2}\right)$ or $L\left(x^{2}\right) L(x)$. In the same manner, again noting that (6) and the choice of $r$ imply that $r_{j-1}$ is minimal, we can write (10) in the form $\alpha z R\left(x^{m}\right) L\left(x^{n_{j}}\right)^{r_{j}} I_{j}$. By the choice of $r$ we have $n_{j} \geqq N$ for large enough $j$ and this $T^{k}=0$.

We denote by $M(A)$ the associative algebra generated by the right and left multiplications of $A$. If $B$ is any subalgebra of $A$ then $B^{*}$ is the subalgebra of $M(A)$ generated by the right and left multiplications of $B$, 
that is, every element in $B^{*}$ is a linear combination of terms of the form $S_{1} S_{2} \cdots S_{n}$ where each $S_{i}$ is a right or left multiplication by an element in $B$.

THEOREM 2. Any finite-dimensional nil algebra A over F satisfying (1) is nilpotent.

Proof. As in the proof that any alternative nil algebra of finite dimension is nilpotent [3, p. 30], we let $B$ be a subalgebra of $A$ which is maximal with respect to the property that $B^{*}$ is nilpotent. Since the subalgebra $\{0\}$ has the property and $A$ is finite dimensional, such a maximal $B$ exists. We assume that $B$ is a proper subalgebra of $A$ and so there exists an element $x$ not in $B$ such that

$$
x B^{*} \subseteq B .
$$

We let

$$
C=B+F[x]
$$

so that $C^{*}=(B+F[x])^{*}$. We will show that $C^{*}$ is a nil algebra. Let $T \in C^{*}$. From (5) we can systematically pass all the right multiplications to the left and from (4) and (11) we can assume that each right multiplication is in $B^{*}$. Note that in this new expression for $z T$ the number of factors from $B^{*}$ is preserved. Hence we can assume that $z T$ is of the form

$$
\alpha z R\left(b_{1}\right) R\left(b_{2}\right) \cdots R\left(b_{r}\right) L\left(a_{1}\right) L\left(a_{2}\right) \cdots L\left(a_{s}\right)
$$

where the $a_{i} \in B$ or $F x, \alpha \in F$ and $z \in A$.

Now from (3) we can replace any $L(x) L(b)$ for $b \in B$ by a linear combination of terms of the form $L(b) L(x), R(x b), R(b x), L(b x), L(x b)$, $L(x) R(b), L(b) R(x), R(x) R(b), R(b) R(x), R(x) L(b)$ and $R(b) L(x)$. Utilizing (4), (5) and (11) allows us to replace $L(x) L(b)$ by a linear combination of terms of the form $L(b) L(x), R\left(b_{1}^{\prime}\right), L\left(b_{2}^{\prime}\right), R(x) L\left(b_{3}^{\prime}\right), R\left(b_{4}^{\prime}\right) L(x)$. As before, all the right multiplications can be passed to the left and each $R(x)$ is enveloped by an $R(b)$. Hence for any $T$ in $C^{*}, z T$ can be written as a linear combination of terms of the form

$$
\alpha z R\left(b_{1}\right) R\left(b_{2}\right) \cdots R\left(b_{r}\right) L\left(b_{r+1}\right) \cdots L\left(b_{s}\right) L(x)^{i}
$$

or of the form

$$
\alpha z R\left(x^{t}\right) L\left(b_{1}\right) L\left(b_{2}\right) \cdots L\left(b_{s}\right) L(x)^{i}
$$

where the number of factors from $B^{*}$ is constant. If $B^{* N}=0$ and $x^{m}=0$, we have $T^{2 n m \bar{m}}=0$, where $\bar{m}$ is the index of nilpotency of the ring mentioned in Theorem 1, because we have either $n$ factors from $B^{*}$ on the left or an element from $\{L(x)\}^{\bar{n}}=0$, from Theorem 1. Hence every element of the 
finite-dimensional associative algebra $C^{*}$ is nilpotent, and so $C^{*}$ is nilpotent [1, p. 23]. But $B$ was maximal with respect to the property of having $B^{*}$ nilpotent, so we have a contradiction, implying that $B$ is not a proper subalgebra of $A$. Thus $A=B$ and $A^{*}$ is nilpotent. It follows [3, p. 18] that $A$ is nilpotent.

\section{REFERENCES}

1. A. A. Albert, Structure of algebras, Amer. Math. Soc. Colloq. Publ., vol. 24, Amer. Math. Soc., Providence, R.I., 1939.

2. R. Coughlin and M. Rich, Associo-symmetric algebras, Trans. Amer. Math. Soc. 164 (1972), 447-451.

3. R. D. Schafer, An introduction to nonassociative algebras, Pure and Appl. Math., vol. 22, Academic Press, New York, 1966. MR 35 \#1643.

Department of Mathematics, Temple University, Philadelphia, Pennsylvania 19122 